\title{
Effects of botulinum toxin injection on systemic sclerosis-related digital ulcers
}

Jin-Woo Souk and Hyun-Sook Kim

Division of Rheumatology, Department of Internal Medicine, Soonchunhyang University Seoul Hospital, Seoul, Korea
Received: June 3, 2018

Revised : June 7, 2018

Accepted: June 11, 2018

\section{Correspondence to}

Hyun-Sook Kim, M.D.

Tel: +82-2-710-3214

Fax: +82-2-709-9554

E-mail: healthyra@schmc.ac.kr
Vasoconstriction caused by paradoxical vascular responses is thought to underlie systemic sclerosis (SSc) related digital ulcer (DU). Recently, botulinum toxin (BTX) has emerged as a chemical sympathectomy. Updated guidelines indicate that BTX injection is a treatment option for severe and refractory Raynaud phenomenon and DU.

Case 1: A 62-year-old female presented with painful DU. She was diagnosed with diffuse cutaneous SSc 10 months prior. As the DU pain was uncontrolled, we injected BTX-type A (10 IU of Meditoxin [Medytox Inc., Seoul, Korea] in $0.1 \mathrm{~mL}$ saline) subcutaneous- ly into the palm, just proximal to the A1 pulley of the left second finger (Fig. 1A). Initially, the fingertip ulcer was $5 \times$ $4 \mathrm{~mm}$ in area, with dark pigmentation (Fig. 1B). After additional injections 4 and 5 weeks later, significant improvements were evident (Fig. $1 \mathrm{C}$ and $1 \mathrm{D}$ ).

Case 2: A 50-year-old SSc female was taking endothelin receptor antagonist to treat the recurrent active DU. At the first visit, a deep ulceration $3 \times 3 \mathrm{~mm}$ in area was evident (Fig. 2A). We injected BTX-type A as described above for case 1. Four days later, the ulcer had improved, but another ulcer had developed (Fig. 2B). We administered
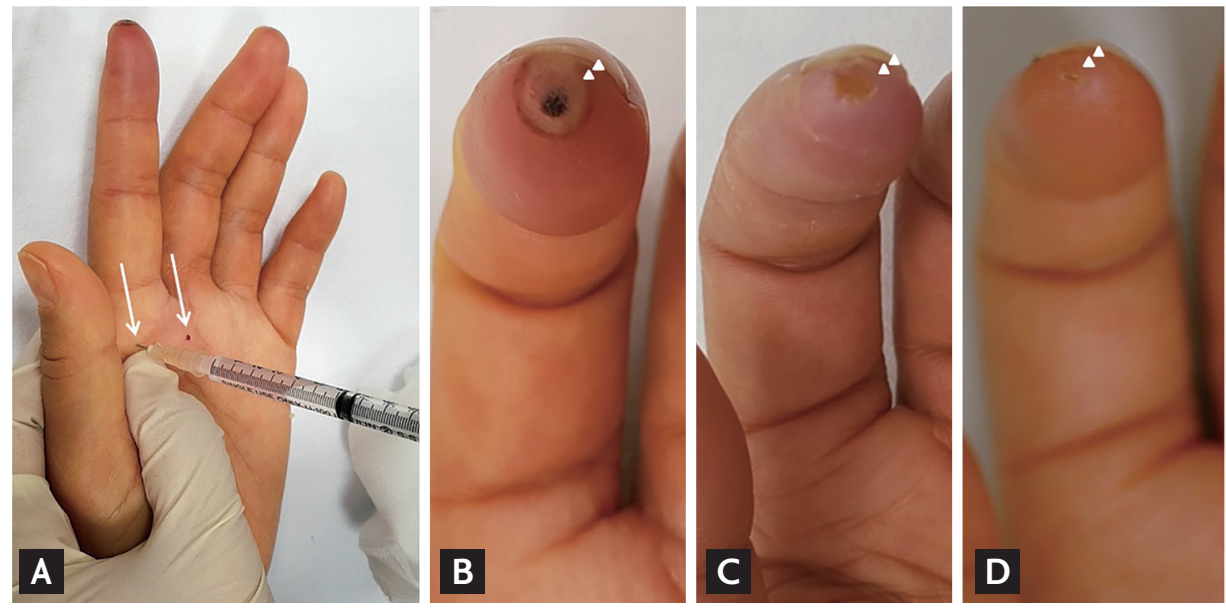

Figure 1. Botulinum toxin (BTX) injection, and serial photographs of digital ulcer. (A) BTX-type A (10 IU) was subcutaneously injected into the palmar aspect of the hand, just proximal to the Al pulley of the left second finger (arrows). (B) At the first visit, the ulcer was $5 \times 4 \mathrm{~mm}$ in area, with black pigmentation. (C) Four weeks after the first BTX injection, the lesional area had decreased to $4 \times 3 \mathrm{~mm}$ and the black pigmentation had disappeared. (D) After two additional BTX injections (at 4 and 5 weeks after the first injection), the ulcer had almost healed. 

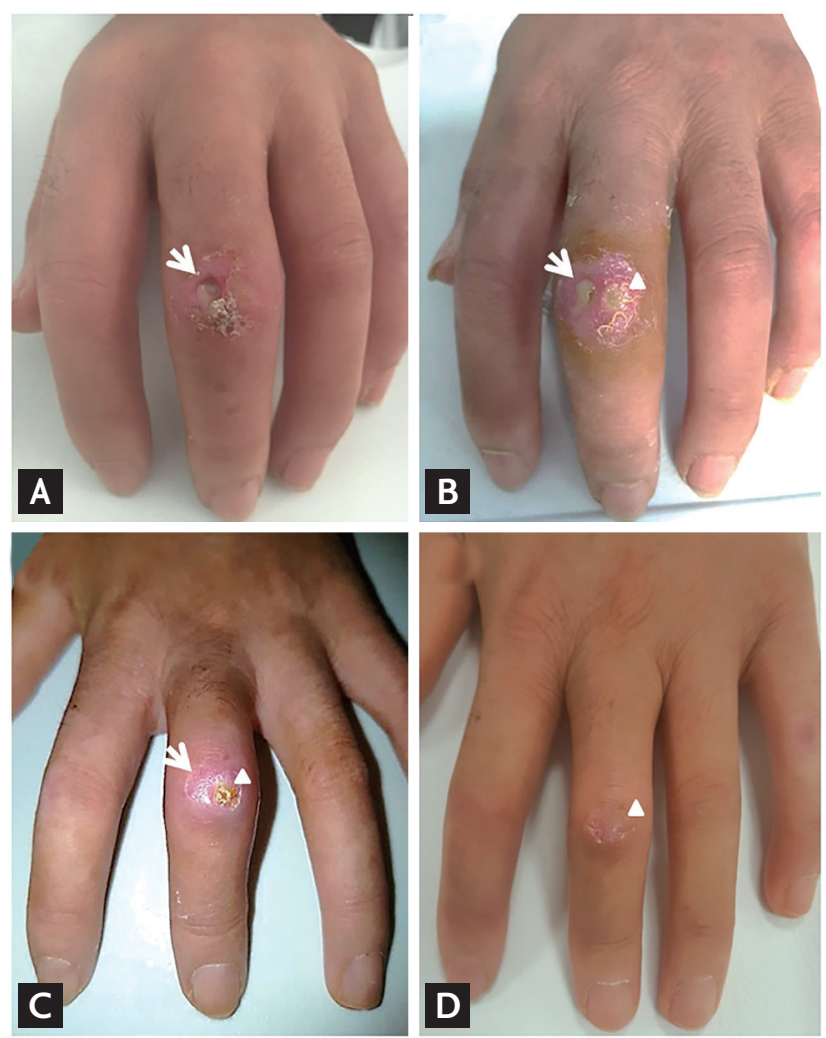

Figure 2. Systemic sclerosis-related, severe digital ulcers (DUs) on the left, third proximal interphalangeal joint healed after multiple botulinum toxin (BTX) injections. (A) At the first visit, a painful ulcer was deep and $3 \times 3 \mathrm{~mm}$ in area, with peripheral redness (arrow). (B) Five days after the first BTXtype A injection, another ulcer (arrowhead) developed just beside the first DU (arrow). (C) Seven weeks after the first BTX injection, the first DU had healed (arrow) and the second DU had improved (arrowhead). (D) After three BTX injections (additional injections were given 7 and 8 weeks after the first injection), the second DU also healed (arrowhead). further BTX injections 7 and 8 weeks after the first injection. Although there was transient exacerbation of the DU caused by wound infection, gradual improvement was evident (Fig. 2C and 2D).

BTX injection is considered as a possible alternative therapy for SSc-related DUs that inhibits the release of acetylcholine and blocks the transmission of the norepinephrine vesicle at the sympathetic nerve fiber, preventing vascular smooth muscle vasoconstriction. BTX may also inhibit endothelial exocytosis of endothelin-1 and increase the activity of nitric oxide. Our patients exhibited improvements in refractory DU lesions after serial BTX injections, but more data are required to verify efficacy and to determine the optimal dose and injection frequency.

This study was approved by the Institutional Review Board of Soonchunhyang University Seoul Hospital (2017-12-015). Written informed consents were obtained.

\section{Conflict of interest}

No potential conflict of interest relevant to this article was reported.

\section{Acknowledgments}

This study is supported by fund of the Soonchunhyang University. 